\title{
Avaliação da eficácia de um fluxograma como auxiliar no ensino de diagnóstico periodontal
}

\author{
Gabriel Crispim Vilar*, Jordana Mendes Schuelter**, Cléverson de Oliveira e Silva***, Flavia Matarazzo \\ Martins****
}

* Mestrando em Odontologia Integrada pela Universidade Estadual de Maringá, UEM.

** Graduação em Odontologia pela Universidade Estadual de Maringá, UEM.

*** Doutor em Clínica Odontológica pela Universidade Estadual de Campinas, UNICAMP-PI.

**** Doutorado em Ciências Biológicas pela Universidade de São Paulo, USP/SP.

\section{RESUMO}

Considerando os vários tipos de doenças periodontais, que requerem diferentes formas de tratamento, é crucial que um diagnóstico preciso seja estabelecido, para se evitar tratamentos ineficazes. Diante disso, este estudo teve como objetivo avaliar o uso de um fluxograma como auxiliar no diagnóstico das doenças periodontais no ensino. Sessenta alunos do curso de Odontologia da Universidade Estadual de Maringá, igualmente distribuídos entre o $3^{\circ}, 4^{\circ}$ e $5^{\circ}$ anos da graduação, aceitaram participar deste estudo experimental e cruzado. Os estudantes foram submetidos à três questões clínicas periodontais hipotéticas, cujos diagnósticos deveriam ser apresentados primeiramente sem o fluxograma e, em seguida, com a ferramenta auxiliar. Sem o fluxograma, a quantidade de diagnósticos corretos foi de $52 \%$, enquanto com fluxograma, este valor correspondeu a $84 \%$, havendo diferença estatística entre eles $(\mathrm{p}<0,001)$. Não houve diferença no número de diagnósticos corretos e incorretos entre os anos de graduação. Pode-se concluir que o uso do fluxograma como auxiliar no diagnóstico periodontal aumenta o número de diagnósticos periodontais corretos.

Descritores: Diagnóstico Periodontal. Doenças Periodontais. Ensino Odontológico. 


\section{INTRODUÇÃO}

As doenças periodontais constituem um problema de saúde pública, pois atingem uma grande proporção da população, devido ao seu caráter inicial inofensivo ${ }^{1}$. Segundo informativo da Organização Pan-Americana de Saúde (OPAS/OMS) em 2001, entre 50 e 99\% das pessoas ao redor do mundo apresentam tais patologias $^{2}$. No entanto, segundo Gomes Filho et al. $^{3}$, o critério de diagnóstico empregado para definição da doença periodontal influencia os índices de prevalência, podendo variar, na mesma população, entre $20,1 \%$ e $47,2 \%$.

O sistema de Classificação das Condições e Doenças Periodontais foi completamente reformulado durante um encontro internacional promovido pela Academia Americana de Periodontia (AAP), em 1999. A classificação vigente na época exigia alterações devido ao avanço no conhecimento científico sobre a etiologia, patogênese e tratamento das doenças periodontais. Para atender aos critérios estabelecidos pela AAP, o cirurgião-dentista deve estar apto a diagnosticar o estado de saúde e o de doença periodontal, ser capaz de diferenciar os tipos de doenças e, por fim, classificar os diferentes graus de extensão e severidade de cada condição ${ }^{4}$.

Considerando os vários tipos de doenças periodontais que requerem diferentes formas de tratamento, é crucial que um diagnóstico preciso seja estabelecido, para se evitar tratamentos ineficazes. Diversas formas de facilitar $o$ diagnóstico periodontal são sugeridas na literatura, tais como, sondas periodontais eletrônicas padronizadas $^{5}$, testes microbiológicos ${ }^{6}$ e análise salivar ${ }^{7}$. No entanto, todas elas refletem em um custo adicional, muitas vezes inacessível, ao paciente e ao profissional. Ferramentas alternativas, mais simples e eficazes, devem ser desenvolvidas no cumprimento deste papel.

Desta maneira, o objetivo deste trabalho foi avaliar a eficácia de um fluxograma baseado no sistema de classificação das Condições e Doenças Periodontais ${ }^{8}$ como auxiliar no ensino da tomada de diagnóstico clínico periodontal em três situações clínicas hipotéticas.

\section{METODOLOGIA}

Um fluxograma para diagnóstico das condições e doenças periodontais (figura 1) foi desenvolvido com base na Classificação das Condições e Doenças Periodontais da Academia Americana de Periodontia, em 1998. Os sintomas, as características clínicas e os fatores relacionados a cada condição periodontal foram organizados de maneira a direcionar o clínico ao correto diagnóstico.

Após a apreciação e aprovação do projeto pelo Comitê de Ética da Universidade Estadual de Maringá (CAAE 06734312.9.0000.0104), acadêmicos do Curso de Odontologia, que já haviam iniciado suas atividades clínicas, foram convidados a participar do estudo. $\mathrm{O}$ curso nesta instituição tem duração de 5 anos e é composto por cerca de 200 estudantes (40 alunos/ano de graduação). As atividades clínicas se iniciam já no primeiro semestre do terceiro ano com a Clínica de Periodontia e continuam durante o quarto e quinto anos em atividades intra e extramuros, respectivamente. Desta maneira, 60 de um total de 120 alunos que cursavam o $3^{\circ}, 4^{\circ} \mathrm{e}$ $5^{\circ}$ anos de graduação aceitaram participar deste estudo experimental e cego. Os participantes assinaram e autorizaram a sua participação por meio do Termo de Consentimento Livre e Esclarecido.

No segundo semestre do ano letivo, os alunos participantes foram submetidos à três questões periodontais hipotéticas, cujos diagnósticos, em diferentes graus de dificuldade (baixo, médio e alto), deveriam ser apresentados.

O primeiro caso clínico tinha como diagnóstico "periodontite ulcerativa necrosante" e foi caracterizado como nível médio de dificuldade, pois requer discernimento entre gengivite e periodontite, além do reconhecimento de características de uma patologia necrosante.

A segunda situação clínica hipotética tinha como diagnóstico "periodontite crônica generali- 


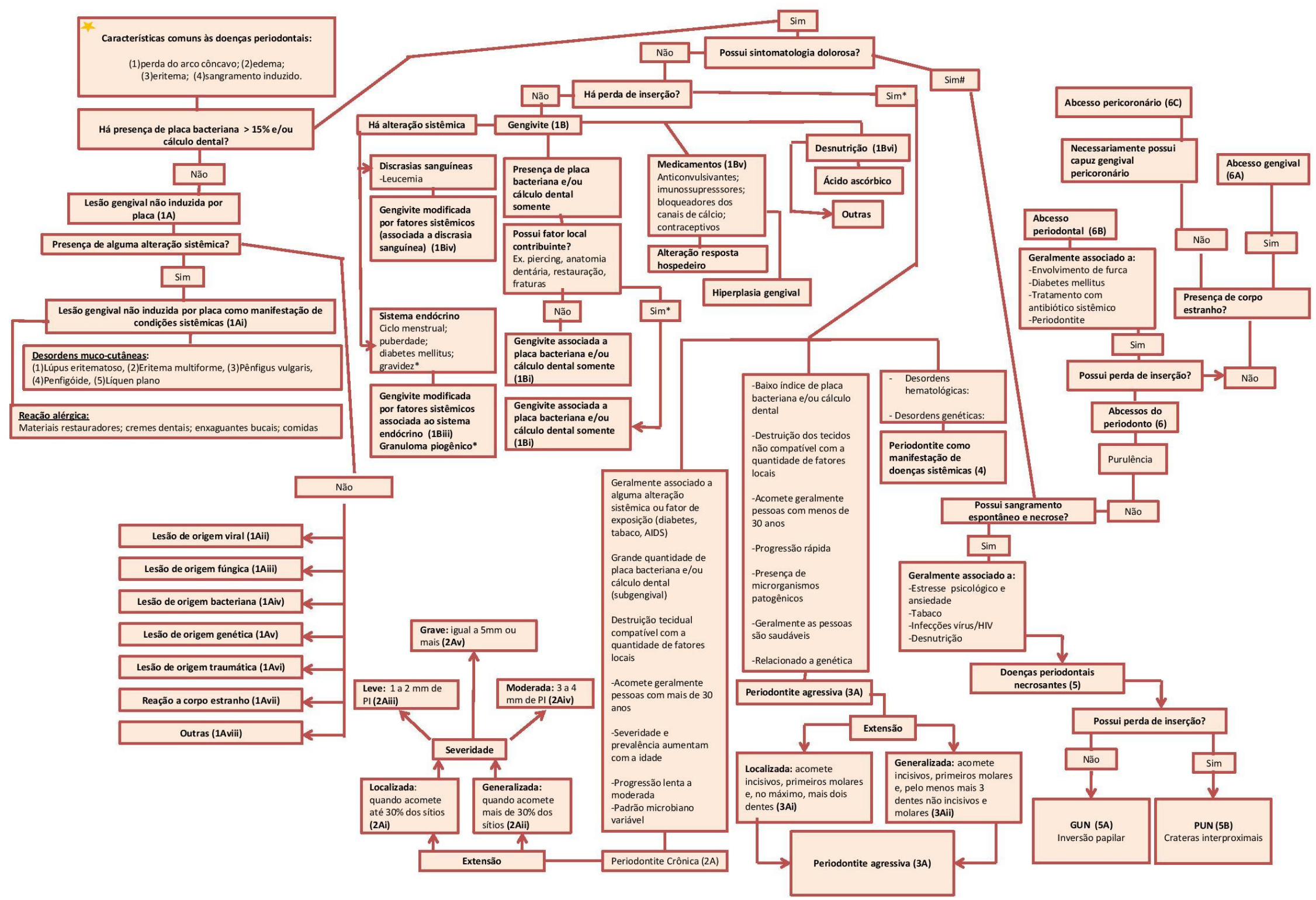

Figura 1 - Fluxograma para diagnóstico das condições e doenças periodontais. 
zada grave", que foi caracterizada como nível baixo de dificuldade, já que exige somente o conhecimento em relação à diferenciação entre gengivite e periodontite. Além disso, a incidência desta doença periodontal é alta nas clínicas, fato que possibilita uma vivência maior deste diagnóstico.

O terceiro caso clínico apresentava o diagnóstico de "lesão gengival não induzida por placa bacteriana como manifestação de alterações sistêmicas relacionada à alteração alérgica creme dental", caracterizada como alto nível de dificuldade, pois exige um conhecimento mais aprofundado das diferentes condições periodontais e não é um caso corriqueiro na clínica diária.

Inicialmente os participantes deram o diagnóstico de cada caso com base no conhecimento adquirido durante os respectivos anos de graduação. Logo em seguida, o fluxograma foi fornecido como método auxiliar para análise dos mesmos casos. Todos os alunos participaram da avaliação com e sem o uso do fluxograma. Apenas ao final de todo o processo, foi revelado o diagnóstico correto de cada caso.

Foram considerados acertos quando o diagnóstico estava de acordo com a classificação da $\mathrm{AAP}^{8}$. O total de questões corretas e incorretas, com e sem o uso do fluxograma foi, então, transferido para o programa BioEstat ${ }^{\circledR}$ (Belém, Brasil). $\mathrm{O}$ efeito do uso do fluxograma no índice de acertos foi analisado utilizando o teste Exato de Fisher.

Para verificar a diferença entre as séries foi utilizado o teste do Qui-quadrado. O nível de significância estatística foi estabelecido em $5 \%$.

\section{RESULTADOS}

O número total de diagnósticos corretos sem o fluxograma foi de $52 \%(\mathrm{n}=93)$ e com a ferramenta auxiliar foi de $84 \%(n=151)$, com diferença estatística entre eles ( $\mathrm{p}<0,001)$ (gráfico 1).

A tabela 1 apresenta a distribuição do número de diagnósticos corretos e incorretos de acordo com o ano de graduação, com e sem a utilização do fluxograma. Sem o fluxograma, o quarto ano apresentou uma maior quantidade de diagnósticos corretos $(n=37)$ comparado ao terceiro e quinto anos, que apresentaram, cada um, 28 acertos. Com o fluxograma, por outro lado, a quantidade de acertos foi semelhante entre os anos de graduação. Não houve diferença estatisticamente significante quando o número de diagnósticos corretos e incorretos foi comparado entre os diferentes anos de graduação, com e sem o fluxograma.

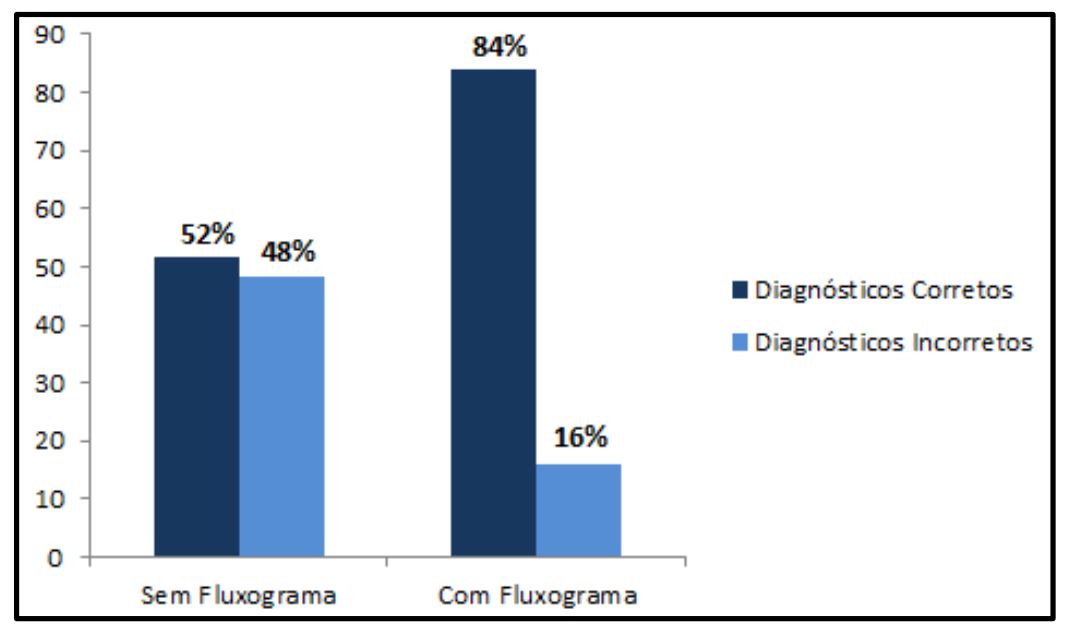

Gráfico 1. Porcentagem (\%) de diagnósticos corretos e incorretos, sem e com o uso do fluxograma.

$*$ Teste Exato de Fisher $(\mathrm{p}<0,001)$ 
Tabela 1 - Distribuição do número de diagnósticos corretos e incorretos de acordo com cada ano, com e sem a utilização do fluxograma.

\begin{tabular}{cccccccc}
\hline & \multicolumn{2}{c}{ Sem Fluxograma } & \multicolumn{2}{c}{ Com Fluxograma } & \\
& $\mathbf{3}^{\mathbf{0}}$ ano & $\mathbf{4}^{\mathbf{0}}$ ano & $\mathbf{5}^{\mathbf{0}}$ ano & $\mathbf{3}^{\mathbf{0}}$ ano & $\mathbf{4}^{\mathbf{0}}$ Ano & $\mathbf{5}^{\mathbf{0}}$ ano & $\boldsymbol{p}$ \\
\hline Diagnósticos Corretos & 28 & 37 & 28 & 52 & 51 & 48 & \\
Diagnósticos Incorretos & 32 & 23 & 32 & 8 & 9 & 12 & $>0,05$ \\
Total & 60 & 60 & 60 & 60 & 60 & 60 & \\
\hline
\end{tabular}

*Teste Qui-quadrado (p>0,05)

\section{DISCUSSÃO}

Até onde sabemos não existe nenhum trabalho que propôs o uso de um fluxograma como auxiliar no ensino do diagnóstico periodontal. Os resultados mostraram que o uso do fluxograma aumentou a quantidade de diagnósticos corretos, entre acadêmicos, independente da série que estava sendo cursada.

A comparação com outros estudos, que também avaliaram a performance de fluxogramas quanto à capacidade de detectar a presença de doenças ou condições, demonstrou que esta ferramenta nem sempre é considerada a chave do sucesso na tomada diagnóstica. Um estudo realizado por Menezes e Faúndes ${ }^{9}$ que buscou validar o uso de um fluxograma para diagnóstico de afecções cérvicovaginais em gestantes mostrou baixa especificidade, sensibilidade e valores preditivos fracos para as doenças avaliadas. A abordagem simplista dos sinais e sintomas das afecções avaliadas e o uso de exames laboratoriais pouco específicos e confiáveis limitaram a segurança do fluxograma no direcionamento diagnóstico.

Segundo Pihlstrom ${ }^{10}$, um diagnóstico periodontal preciso só pode ser feito com uma avaliação completa dos dados do paciente que devem ser sistematicamente recolhidos através da anamnese, consulta médica, quando necessário, exame clínico periodontal, radiografias e exames laboratoriais. As três situações clínicas hipotéticas foram criadas oferecendo informações sobre $o$ estado sistêmico do paciente, características relacionadas à saúde bucal e principalmente periodontal. Como não foram oferecidas imagens radiográficas aos alunos, as características relevantes foram descritas. Para a resolução destes casos clínicos não havia necessidade de informações de exames laboratoriais por isso não foram mencionados, não descartando a necessidades destes exames em casos que requerem uma abordagem mais ampla.

A ausência de diferença significativa entre as séries pode indicar que, independente do grau de formação e do conhecimento adquirido através dos anos, o fluxograma se mostrou eficaz como auxiliar na tomada diagnóstica. Cada decisão ou ação leva a uma ou mais rotas que, por sua vez, levam a um quadro com outra decisão ou ação ${ }^{11}$, 12. Desta maneira, o fluxograma tem o potencial de auxiliar cirurgiões-dentistas clínicos gerais, a se direcionarem de maneira rápida e eficaz durante o diagnóstico periodontal. Este efeito seria benéfico, por exemplo, no encaminhamento de pacientes atendidos em unidades básicas de saúde para centros especializados. Muitas vezes pacientes de baixo risco para progressão da doença periodontal, ou seja, com gengivite, que poderiam ser tratados no próprio posto de saúde inflam as listas de espera de centros especializados, retardando o tratamento dos casos mais severos, que se agravam com o tempo ${ }^{11}$.

Existem algumas limitações em relação ao número de participantes e ao tempo clínico de 
atuação da população analisada. Apesar destas limitações, apenas com estes dados, já foi possível observar que o fluxograma se mostra eficiente na utilização como método auxiliar na tomada diagnóstica. Mais estudos devem ser realizados abrangendo profissionais da rede pública e privada para validar sua efetividade.

\section{CONCLUSÃO}

O fluxograma desenvolvido para ser usado como ferramenta auxiliar no ensino de diagnóstico periodontal mostrou-se eficiente para atingir tal objetivo. Em casos clínicos menos comuns e com maior grau de dificuldade o fluxograma mostrouse mais eficaz.

\section{ABSTRACT \\ Evaluation of the effectiveness of a flowchart as an adjunct in teaching periodontal diagnosis}

Considering the different types of periodontal diseases, which require different forms of treatment, the establishment of an accurate diagnosis is critical, to avoid inefficient treatments. Thus, this study aimed to evaluate the use of a flow chart as an aid in the teaching diagnosis of periodontal disease. Sixty students of Dentistry at the Universidade Estadual de Maringá, equally distributed between the 3rd, 4th and 5th year graduation accepted to participate to this experimental and crossover study. Students underwent three hypothetical periodontal clinical questions, whose diagnoses should first be presented without the flowchart and then with the auxiliary tool. Without the flowchart, the amount of correct diagnosis was $52 \%$, while with the flowchart, this value corresponded to $84 \%$ (p $<0.0001)$. There was no difference in the number of correct and incorrect diagnoses between the years of graduation. It can be concluded that the use of the flowchart as an aid in diagnosing periodontal increases the number of correct periodontal diagnosis.

Descriptors: Periodontal Diagnosis. Periodontal Diseases. Dental, Teaching.

\section{REFERÊNCIAS}

1. Goulart JHM, Santos Júnior JC, Silva L, Souza LMA. Um Sistema para triagem de pacientes com doenças periodontais. In: VI Congresso de Iniciação Científica da UFS, 2004, Aracaju. Anais do VI Congresso de Iniciação Científica da UFS, 2004. p. 22-22.

2. Organização Pan-Americana de Saúde. Saúde Bucal. Acesso em 4/12/2015. Disponível em: http://www.uff.br/farmacobasica-mfl/sites/de fault/files/saude bucal.pdf.

3. Gomes Filho IS, Macedo TCN, Cruz SS, Soledade KR, Trindade SC, Sarmento VA. Comparação de critérios que determinam o diagnóstico clínico da doença periodontal. Odonto Ciência 2006; 21:77-81.

4. Williams RC, Paquette DW. Avanços no diagnóstico periodontal, em: Lindhe, J, Karring T, Lang NP -Tratado de Periodontia Clínica e Implantologia Oral. $3^{\mathrm{a}} \mathrm{Ed}$, Rio de Janeiro: Guanabara Koogan, 1999; p. 281-98.

5. Ference R. Diagnosis of periodontal diseases. Changing concepts. A challenge for the future. N Y State Dent J 1989;55(6):30-3.

6. D'Ercole S, Catamo G, Piccolomini R. Diagnosis in periodontology: a further aid through microbiological tests. Crit Rev Microbiol 2008; 34(1):33-41.

7. Kaufman E, Lamster IB. Analysis of saliva for periodontal diagnosis - a review. J Clin Periodontol 2000; 27(7):453-65.

8. Armitage GC. Development of a classification system for periodontal diseases and conditions. Ann Periodontol 1999; 4:1-6.

9. Menezes MLB, Faúndes AE. Validação do fluxograma de corrimento vaginal em gestantes. DST J Bras Doenças Sex Transm 2004; 16(1), 38-44.

10. Pihlstrom BL. Periodontal risk assessment, diagnosis and treatment planning. Periodontology 2000 2001; 25:37-58.

11. Atuação do enfermeiro no diagnóstico e no tratamento do herpes genital, na atenção primária à saúde. Acesso em: 17/11/2015. 
Disponível em: http://www.convibra.com.br/ artigo.asp? ev $=24 \& p=\&$ lang $=p t \& i d=7841$

12. Faris AA. Yaser A. Zaid A. Omar K. The Differential diagnosis of desquamative gingivitis: review of the literature and clinical guide for dental undergraduates. Journal of International Oral Health 2015; 7(Suppl 1):88-92.
Correspondência para:

Profa. Dra. Flavia Matarazzo Martins

e-mail: flamatarazzo@gmail.com

Av. Mandacaru, 1550

Campus Universitário

87080-000 Maringá, PR 